\title{
Glückwunsch für Prof.W. Burckhardt
}

Zum 60. Geburtstag möchten wir Ihnen im Namen Ihrer Freunde, Kollegen, Schüler und von zahlreichen medizinischen Gesellschaften, deren Vorstandsmitglied Sie waren, herzlich gratulieren. Die inter-nationale Anerkennung Ihrer unermüdlichen wissenschaftlichen Tätig-keit mit erfolgreicher Forschung besonders auf dem Gebiet des Ek-zems, der Lichtdermatosen, Venereologie und Physio-pathologic dcr Haut möge durch die Beiträge der vorliegenden Festschrift unter-strichen werden. Es ist Ihnen gelungen, mit relativ einfachen, aber klug eingesetzten Mitteln für die wissenschaftliche Erkenntnis und die dermatologische Praxis viele wesentliche Resultate zu erzielen, und dadurch die Achtung und Anerkennung von Kollegen und Studenten zu erwerben. Wir wünschen Ihnen weiterhin Gesundheit und Wohl-ergehen. Möge Ihre aufgeschlossene Persönlichkeit und Ihre schöpfe-rische Tatkraft noch lange der Forschung, der ärztlichen Fortbildung, den Studenten sowie den Patienten erhalten bleiben.

Mit herzlichen Glückwünschen

H. Storck und Mitarbeiter

F. Tenchío und E. Diem mit Kollegen und Freunden

W. Jadassohn und R. Schupplí Redaktion der Dermatologia

$21 \Gamma>$ pi $\cdot$ niatologica, Vol. 130, No. 5 (1965) 